\title{
Potenciais evocados auditivos de tronco encefálico em usuários de crack e múltiplas drogas
}

\author{
Auditory brainstem evoked potentials in crack and multiple \\ drugs addicts
}

\author{
Loretta Fabianni Nigri ${ }^{1}$, Alessandra Giannella Samelli², Eliane Schochat ${ }^{3}$
}

\begin{abstract}
RESUMO
Objetivo: Analisar os potenciais evocados auditivos de tronco encefálico em usuários de crack e múltiplas drogas, bem como levantar as possíveis queixas auditivas e de equilíbrio nesta população. Métodos: Foram avaliados 40 usuários de drogas (20 com uso há mais de cinco anos e 20 há menos de cinco anos) e 20 não usuários, do sexo masculino, com idades entre 19 e 46 anos, com limiares auditivos dentro da normalidade. Resultados: Não houve diferenças significativas dos potenciais evocados auditivos de tronco encefálico dos usuários de drogas quando comparados ao grupo controle. Conclusão: Os potenciais evocados auditivos de tronco encefálico dos usuários de drogas não diferiram significantemente do grupo controle. As queixas apresentadas pelos usuários de drogas foram hiperacusia, alucinação auditiva, zumbido e alteração de equilíbrio.
\end{abstract}

Descritores: Audição; Potenciais evocados auditivos do tronco encefálico; Efeitos de drogas; Cocaína/efeitos adversos; Cocaína crack/efeitos adversos; Drogas ilícitas/efeitos adversos

\section{INTRODUÇÃO}

O consumo de substâncias psicoativas tem gerado, em todas as partes do mundo, problemas sociais e de saúde pública de grande importância, devido a sua crescente prevalência ${ }^{(1-3)}$.

A cocaína é um alcalóide extraído da folha da Erythroxylon coca; pode ser encontrada em duas formas: sal de hidrocloreto e "base livre". A primeira forma pode ser utilizada por via oral, injetada ou inalada; já a "base livre" é a mistura da cocaína com a amônia e o bicarbonato de sódio, que é usada para fumar ( crack), sendo considerada a forma mais potente da droga ${ }^{(4)}$.

A cocaína, quando inalada, é absorvida rapidamente pelos vasos pulmonares e atinge a circulação cerebral em aproximadamente seis a oito segundos, produzindo intensa euforia. As maiores concentrações da droga são encontradas no cérebro, no baço, nos rins e no pulmão ${ }^{(4)}$.

Pesquisas têm mostrado que a cocaína promove alterações

Trabalho realizado no Curso de Fonoaudiologia da Faculdade de Medicina da Universidade de São Paulo - USP - São Paulo (SP), Brasil.

(1) Mestre, Fonoaudióloga clínica - São Paulo (SP), Brasil.

(2) Doutora, Professora do Curso de Fonoaudiologia da Faculdade de Medicina

da Universidade de São Paulo - USP - São Paulo (SP), Brasil.

(3) Livre Docente, Professora Associada do Curso de Fonoaudiologia da Faculdade de Medicina da Universidade de São Paulo - USP - São Paulo (SP), Brasil.

Endereço para correspondência: Alessandra Giannella Samelli. R.

Cipotânea, 51, Cidade Universitária, São Paulo (SP), Brasil, CEP: 05360-000.

E-mail: alesamelli@usp.br

Recebido em: 5/11/2008; Aceito em: 3/3/2009 degenerativas nos neurônios e células gliais, assim como modificações nas funções dos receptores, na produção e na liberação de alguns neurotransmissores. Contudo, os processos neuroquímicos e vasculares envolvidos nesta aparente neurotoxicidade não foram ainda esclarecidos totalmente ${ }^{(3)}$.

O uso de drogas por mulheres grávidas é um assunto bem conhecido e estudado. Sabe-se que o uso de drogas materno tem efeito no desenvolvimento do cérebro fetal, podendo ocorrer de duas formas principais: nas primeiras 20 semanas de gestação, atuando sobre a citogênese e a migração celular; na segunda metade da gestação, atuando sobre o crescimento do cérebro e sua diferenciação. A cocaína tem livre acesso na barreira placentária e pode causar hipóxia fetal em virtude da vasoconstrição placentária. Além disso, estudos animais mostraram alterações na organização e migração de neurônios e células da glia ${ }^{(5)}$.

A cocaína utilizada durante a gestação pode alterar também o sistema auditivo. Crianças expostas à cocaína durante o período pré-natal mostraram latências absolutas e interpicos prolongados referentes aos Potenciais Evocados Auditivos de Tronco Encefálico (PEATE), no primeiro ano de vida. Estas mesmas alterações eletrofisiológicas foram observadas em ratos, além do aumento dos limiares eletrofisiológicos ${ }^{(6-7)}$. Estas alterações podem ser causadas por falhas na função dos neurotransmissores ou por imaturidade mielínica; a hipóxia também pode contribuir para este aumento das latências, uma vez que o tronco encefálico é particularmente sensível à privação de oxigênio ${ }^{(6)}$. 
Para o usuário de drogas, o local de ação da cocaína no sistema auditivo pode incluir estruturas periféricas. Estudos prévios sobre os efeitos agudos da cocaína demonstraram uma diminuição no fluxo sangüíneo da cóclea, que pode causar uma diminuição na amplitude dos potenciais de ação do nervo $\operatorname{auditivo}^{(8-9)}$.

Embora existam numerosos estudos a respeito dos efeitos danosos da cocaína sobre o sistema auditivo (periférico ou central) em neonatos ou cobaias expostos às drogas durante o período pré-natal, pesquisas sobre este assunto, com indivíduos adultos que fizeram uso de drogas por longos períodos, são escassas.

Sendo assim, os objetivos deste estudo foram analisar os potenciais evocados auditivos de tronco encefálico em usuários de crack e múltiplas drogas, bem como levantar as possíveis queixas auditivas e de equilíbrio nesta população.

\section{MÉTODOS}

A casuística foi composta por três grupos de indivíduos do sexo masculino, na faixa-etária entre 19 e 46 anos, sendo:

- Grupo A: 20 indivíduos não usuários de drogas (grupo controle);

- Grupo B: 20 indivíduos usuários de drogas há mais de cinco anos;

- Grupo C: 20 indivíduos usuários de drogas há menos de cinco anos.

Os indivíduos que compuseram os três grupos foram pareados de acordo com a idade. $\mathrm{O}$ grupo controle foi selecionado dentre os residentes e funcionários Instituto de Psiquiatria do Hospital das Clínicas da Faculdade de Medicina da Universidade de São Paulo (FMUSP). Já os grupos B e C foram recrutados em duas instituições públicas para a reabilitação de usuários de drogas: no ambulatório do Grupo Interdisciplinar de Estudos de Álcool e Drogas (GREA) do Instituto de Psiquiatria do Hospital das Clínicas da FMUSP, e no Centro de Reabilitação de Usuários de Drogas de Santana.

Todos os indivíduos dos três grupos deveriam apresentar limiares auditivos e análise das funções de orelha média dentro dos limites de normalidade. Como critério de exclusão para participação da pesquisa foi considerado a presença de qualquer distúrbio emocional, cognitivo ou psiquiátrico que incapacitasse o indivíduo de compreender as informações dadas durante a obtenção do Termo de Consentimento Livre e Esclarecido (TCLE). Para os grupos B e C, o critério de inclusão primordial foi o diagnóstico de dependência de drogas ilícitas ou ter tido experiência com uso de crack/cocaína.

A pesquisa teve início após a aprovação do projeto de pesquisa pela Comissão de Ética para Análise de Projetos de Pesquisa (CAPPesq) do Hospital das Clínicas da FMUSP sob o número 787/00.

A seleção dos indivíduos foi feita a partir da análise de prontuários das instituições nas quais os dados foram coletados e por meio de anamnese aplicada nos mesmos locais. Os participantes, após convenientemente esclarecidos, caso concordassem, deveriam assinar o TCLE.

O questionário adaptado do Addiction Severity Index $(\mathrm{ASI})^{10)}$ foi aplicado a todos os participantes, obtendo in- formações sobre histórico médico, tratamentos realizados e histórico do uso de drogas/álcool (tipo, tempo de uso, via de administração, abstinência e tratamentos), assim como histórico psiquiátrico (Anexo 1). Este questionário costuma ser utilizado por psiquiatras com usuários de drogas. Também foi aplicada uma anamnese audiológica com adição de algumas perguntas referentes ao uso de drogas.

Em seguida, foram realizados os seguintes procedimentos:

- Audiometria tonal liminar: determinando os limiares por via aérea nas frequências de 250 a 8000 Hz. O audiômetro utilizado foi o Madsen ${ }$, modelo ZS 76 - IB, com fones TDH 39P. Todos os sujeitos apresentaram limiares auditivos menores do que 20 dBNA em todas as frequências avaliadas.

- Imitanciometria (timpanometria e pesquisa dos reflexos acústicos): o equipamento para a avaliação foi o Madsen ${ }$, modelo ZS 76 - IB. Com este procedimento buscou-se eliminar quaisquer comprometimentos de orelha externa e/ou média, os quais poderiam influenciar os achados da pesquisa. Antes da realização da imitanciometria, todos os indivíduos passaram por uma meatoscopia, confirmando a ausência de cerume ou de outros fatores que pudessem impedir a efetivação da avaliação. Todos os sujeitos apresentaram curva timpanométrica do tipo A e presença de reflexos acústicos contralaterais ${ }^{(11)}$.

- Potenciais Evocados Auditivos de Tronco Encefálico (PEATE): este exame foi realizado com o indivíduo sentado, imóvel, em local silencioso, para evitar qualquer interferência nos traçados. O equipamento usado foi o modelo TravelerExpress, da marca Biologic®. Foi feita assepsia nos locais de colocação dos eletrodos (mastóides e vértex) e foi utilizada pasta eletrolítica para melhor captação dos potenciais. Os estímulos acústicos foram dados por meio dos fones TDH 39P. Os parâmetros para captação dos potenciais foram: impedância dos eletrodos abaixo de 5 omhs, 2000 cliques com polaridade rarefeita, velocidade de 19 cliques/segundo, intensidade de 80 dBNA, amplitude com sensibilidade de $50 \mu \mathrm{V}$, filtros de $100 \mathrm{a}$ $1500 \mathrm{~Hz}$, tempo de análise de $10 \mathrm{~ms}$. Foram analisadas as ondas I, III e V, bem como os intervalos interpicos I-III, III-V e I-V.

Para a análise estatística, foi aplicado o teste ANOVA (Análise da Variância simples, que é utilizado para análise de experiências com vários grupos de observações classificados por meio de um só fator), com nível de significância de $5 \%$ ou $\mathrm{p}<0,05$.

\section{RESULTADOS}

\section{Caracterização dos sujeitos}

Como pode-se observar na Tabela 1, não houve diferença estatisticamente significante em relação à idade dos partici-

Tabela 1. Dados descritivos referentes à idade dos participantes (em anos) dos três grupos

\begin{tabular}{lccc}
\hline & Grupo A & Grupo B & Grupo C \\
\hline Média & 33,25 & 33,19 & 32,00 \\
Desvio-padrão & 8,38 & 7,35 & 8,56 \\
\hline Valor de p & & 0,852 & \\
\hline
\end{tabular}

Teste Anova simples 
pantes dos três grupos, podendo considerá-los homogêneos. A média de idade dos usuários de drogas (grupos B e C) foi de aproximadamente 32,6 anos.

Em relação ao tempo de uso das drogas, o grupo B teve uma média de 13,3 anos ( $\mathrm{DP}=4,25)$ para a cocaína e 7,75 anos $(\mathrm{DP}=2,26)$ para o crack. Já o grupo C teve média de 3,95 anos $(\mathrm{DP}=1,05)$ para a cocaína e 2,3 anos $(\mathrm{DP}=0,8)$ para o crack.

Todos os usuários de drogas, tanto do grupo B quanto do grupo $\mathrm{C}$, utilizam ou utilizavam outras drogas (lícitas ou ilícitas), além da cocaína e do crack. Incluem-se, neste caso, a maconha, a heroína, o ecstasy, sedativos, analgésicos e álcool.

No que se refere ao tempo de abstinência, o grupo B teve uma média de 94,75 dias ( $\mathrm{DP}=115,05)$, enquanto para o grupo C a média foi de 245,25 dias ( $\mathrm{DP}=326,51)$.

Já no que diz respeito às queixas otológicas pré e pós início do uso de drogas, $80 \%$ dos usuários do grupo B não referiram diferença de audição; somente $20 \%$ referiram alguma modificação, sendo $10 \%$ hiperacusia, $5 \%$ alucinação auditiva e 5\% alteração de equilíbrio. No grupo C, 55\% dos usuários não apresentaram mudanças em relação à audição, pré e pós início do uso de drogas; no entanto, $45 \%$ dos usuários queixaram-se de alguma alteração auditiva: 15\% hiperacusia, $15 \%$ alucinação auditiva, 10\% zumbido e 5\% alteração do equilíbrio.

\section{Potenciais Evocados Auditivos de Tronco Encefálico}

Em relação às Tabelas 2 e 3, pode-se observar que a única análise que apresentou diferença estatisticamente significante entre os três grupos foi a latência da onda $\mathrm{V}$ da orelha esquerda. Todas as outras análises, em relação às latências das ondas I, III e V, nas duas orelhas, não apresentaram diferenças estatisticamente significantes entre os grupos.

Tabela 2. Latências das ondas I, III e V da orelha direita dos três grupos

\begin{tabular}{llllll}
\hline $\begin{array}{l}\text { Ondas } \\
\text { (ms) }\end{array}$ & & Grupo A & Grupo B & Grupo C & $\begin{array}{c}\text { Valor } \\
\text { de } p\end{array}$ \\
\hline Onda I & Média & 1,76 & 1,70 & 1,73 & 0,668 \\
& Desvio-padrão & 0,19 & 0,22 & 0,16 & \\
\hline Onda III & Média & 3,59 & 3,59 & 3,73 & 0,488 \\
& Desvio-padrão & 0,71 & 0,19 & 0,23 & \\
\hline Onda V & Média & 5,62 & 5,68 & 5,86 & 0,487 \\
& Desvio-padrão & 1,13 & 0,29 & 0,18 & \\
\hline
\end{tabular}

Legenda: $\mathrm{ms}=$ milissegundos; Teste Anova simples

No que se refere às Tabelas 4 e 5 não foi observada nenhuma diferença estatisticamente significante na comparação entre os três grupos, considerando os intervalos interpicos I-III, III-V e I-V, para as duas orelhas.

\section{DISCUSSÃO}

De acordo com estudos prévios, a maioria dos usuários de drogas é do sexo masculino ${ }^{(12-14)}$. Este foi o motivo da escolha deste gênero para o presente estudo.

Com relação à idade dos usuários de drogas, obteve-se uma média de 32,6 anos, variando de 19 a 46 anos, que é aproxima-
Tabela 3. Latências das ondas I, III e V da orelha esquerda dos três grupos

\begin{tabular}{llllll}
\hline $\begin{array}{l}\text { Ondas } \\
(\mathrm{ms})\end{array}$ & & Grupo A & Grupo B & Grupo C & $\begin{array}{l}\text { Valor } \\
\text { de } \mathrm{p}\end{array}$ \\
\hline Onda I & Média & 1,75 & 1,74 & 1,70 & 0,670 \\
& Desvio-padrão & 0,19 & 0,22 & 0,23 & \\
\hline Onda III & Média & 3,64 & 3,48 & 3,62 & 0,751 \\
& Desvio-padrão & 0,68 & 0,76 & 0,63 & \\
\hline Onda V & Média & 5,85 & 5,72 & 5,87 & $0,045^{*}$ \\
& Desvio-padrão & 0,15 & 0,13 & 0,18 & \\
\hline
\end{tabular}

Legenda: $\mathrm{ms}=$ milissegundos; Teste Anova simples; ${ }^{*}$ Valor estatisticamente significante

Tabela 4. Intervalos interpicos I-III, III-V e I-V da orelha direita dos três grupos

\begin{tabular}{|c|c|c|c|c|c|}
\hline $\begin{array}{l}\text { Interpicos } \\
\text { (ms) }\end{array}$ & & Grupo A & Grupo B & Grupo C & $\begin{array}{l}\text { Valor } \\
\text { de } p\end{array}$ \\
\hline \multirow{2}{*}{$\begin{array}{l}\text { Interpico } \\
\text { I-III }\end{array}$} & Média & 1,98 & 1,86 & 2,00 & \multirow{2}{*}{0,179} \\
\hline & Desvio-padrão & 0,23 & 0,13 & 0,27 & \\
\hline \multirow{2}{*}{$\begin{array}{l}\text { Interpico } \\
\text { III-V }\end{array}$} & Média & 2,12 & 2,08 & 2,13 & \multirow{2}{*}{0,868} \\
\hline & Desvio-padrão & 0,19 & 0,38 & 0,24 & \\
\hline \multirow{2}{*}{$\begin{array}{l}\text { Interpico } \\
\text { I-V }\end{array}$} & Média & 4,11 & 3,95 & 4,13 & \multirow{2}{*}{0,868} \\
\hline & Desvio-padrão & 0,27 & 0,41 & 0,24 & \\
\hline
\end{tabular}

Legenda: $\mathrm{ms}=$ milissegundos; Teste Anova simples

Tabela 5. Intervalos interpicos I-III, III-V e I-V da orelha esquerda dos três grupos

\begin{tabular}{llllll}
\hline $\begin{array}{l}\text { Interpicos } \\
\text { (ms) }\end{array}$ & Grupo A & Grupo B & Grupo C & $\begin{array}{l}\text { Valor } \\
\text { de } p\end{array}$ \\
\hline Interpico & Média & 2,04 & 1,95 & 2,01 & 0,598 \\
I-III & Desvio-padrão & 0,23 & 0,25 & 0,27 & \\
\hline Interpico & Média & 2,06 & 2,15 & 2,13 & 0,524 \\
III-V & Desvio-padrão & 0,17 & 0,32 & 0,29 & \\
\hline Interpico & Média & 4,09 & 4,01 & 4,14 & 0,360 \\
I-V & Desvio-padrão & 0,25 & 0,34 & 0,25 & \\
\hline
\end{tabular}

Legenda: $\mathrm{ms}=$ milissegundos; Teste Anova simples

da da faixa-etária descrita na literatura. A baixa faixa-etária é explicada pela maior exposição e grande penetração das drogas entre os jovens ${ }^{(12,14)}$.

Segundo uma caracterização ${ }^{(10)}$ de uma amostra de 440 usuários de drogas internados em hospitais psiquiátricos para tratamento da dependência química, a maioria dos usuários era do sexo masculino $(95,9 \%)$, de raça branca $(72,7 \%)$, baixo poder aquisitivo (70\%), baixo nível de escolaridade (50\% não tinham completado a $8^{\mathrm{a}}$ série) e com média de idade de 27,5 anos. Usavam cocaína cheirada 131 adictos (29,8\%), 169 $(38,4 \%)$ eram dependentes de crack e 140 (31,8\%) utilizavamse de ambas as formas da droga (pó e crack). Com relação ao uso de outras drogas associadas, 69\% também usavam álcool, $86,1 \%$ tabaco, $55,9 \%$ usavam maconha, 1,6\% droga por via endovenosa (heroína, anfetaminas ou cocaína endovenosa), $1,1 \%$ solventes (cola de sapateiro, esmalte, "tinner", benzina) e dois usavam heroína na forma de pó. 
Esta diversidade no uso de outras drogas associada ao uso da cocaína e/ou crack também foi verificada no presente estudo, sendo que todos os usuários dos dois grupos faziam uso de, pelo menos, uma outra droga (lícita ou ilícita).

Em relação ao tempo de uso das drogas, ambos os grupos utilizam cocaína há mais tempo que o crack. Este fato pode estar relacionado com o surgimento mais recente do crack (em 1985, nas Bahamas), que se popularizou na década de $90^{(1)}$. Além disso, parece haver um padrão de escalonamento no uso de drogas, iniciando-se pelas mais leves e migrando para as mais pesadas ${ }^{(12)}$, como é o caso do crack, quando comparado à cocaína inalada.

Já no que diz respeito às queixas otológicas, a maioria dos usuários de drogas não referiu diferença de audição pré e pós início do uso de drogas. As alterações relatadas foram: hiperacusia, alucinação auditiva, zumbido e alteração de equilíbrio.

A hiperacusia, que é uma tolerância reduzida a sons, pode estar associada a alterações periféricas do sistema auditivo, disfunções de sistema nervoso central, doenças hormonais e infecciosas, bem como pode apresentar-se como um sintoma isolado, de etiologia desconhecida. Embora os mecanismos fisiopatológicos da hiperacusia sejam muito discutidos, de modo geral, se relacionam a falhas na codificação da intensidade, que pode ocorrer nas vias auditivas periféricas ou centrais ${ }^{(15-16)}$.

Considerando-se que a cocaína promove alterações degenerativas nos neurônios, assim como modificações nas funções dos receptores, na produção e na liberação de alguns neurotransmissores ${ }^{(15)}$, poder-se-ia levantar a hipótese de que, nos casos relatados de hiperacusia, a droga poderia ter causado uma alteração no sistema nervoso central que, de alguma forma, estaria interferindo na codificação de intensidade, dando origem ao referido sintoma, nestes indivíduos.

Da mesma forma, a produção do zumbido pode envolver diversas estruturas auditivas periféricas e/ou centrais, bem como a participação de outros sistemas não-auditivos ${ }^{(17-18)}$. Como no caso da hiperacusia, uma possível alteração no sistema nervoso central, causada pela droga, pode ter iniciado a produção do zumbido.

No que se refere às queixas de alteração do equilíbrio, pode-se dizer que o número encontrado entre os usuários de drogas é pequeno, o que está de acordo com achados anteriores $^{(19)}$, quando foram estudados 51 usuários de crack utilizando a vecto-electronistagmografia, dentre os quais nenhum usuário com vestibulometria alterada $(66,6 \%)$ referiu quaisquer sintomas supostamente vestibulares durante a anamnese. Como hipótese para tal ocorrência, foram sugeridas dificuldade na recordação da ocorrência dos sintomas e auto-compensação labiríntica em lesões vestibulares lentamente progressivas ${ }^{(19)}$.

Considerando o fato de que várias drogas têm a capacidade de desencadear sintomas psicóticos como alucinações ou delírios, parece plausível que elas possam iniciar ou causar um episódio esquizofrênico ${ }^{(14,20)}$. Sendo assim, a ocorrência de alucinações auditivas entre quatro usuários de drogas, mesmo no período de abstinência, deve ser considerada e investigada durante o processo de reabilitação destes indivíduos.

Alguns estudos vêm pesquisando os efeitos do uso crônico da cocaína sobre a função cerebral e têm demonstrado a possibilidade do desenvolvimento de complicações psiquiátricas ou neurológicas. Estes trabalhos mostraram uma diminuição das amplitudes e aumento das latências dos potenciais evocados auditivos de média e longa latência entre usuários de drogas, mesmo em períodos de abstinência, o que também é observado em pacientes esquizofrênicos/com transtornos psiquiátricos e em pessoas que mostraram uma maior chance de desenvolverem problemas com drogas e álcool. Os achados alterados dos potenciais evocados auditivos de média e longa latência, nestes casos, sugerem uma inabilidade de filtrar estímulos sensoriais não relevantes, de atenção seletiva, de manutenção da memória de trabalho e de discriminação, bem como foram associados com a existência de maior impulsividade ${ }^{(21-23)}$.

A análise das latências absolutas e interpicos dos potenciais evocados auditivos de tronco encefálico (PEATE) feita no presente estudo não revelou diferenças entre os grupos de usuários de drogas e o grupo controle. O único parâmetro que mostrou diferença estatisticamente significante foi a latência da onda $\mathrm{V}$ da orelha esquerda, com menor resposta para o grupo de usuários de drogas há mais de cinco anos. Desta forma, pode-se considerar que, no geral, não houve diferenças marcantes dos PEATE entre os grupos.

Tal achado sugere que, em nível de tronco encefálico, as drogas não provocaram alterações evidentes, as quais pudessem comprometer as ondas dos PEATE. Isto não significa que os usuários de drogas não tenham nenhuma lesão/disfunção nas estruturas que fazem parte das vias auditivas, já que as estruturas acima do tronco encefálico não foram avaliadas. É importante salientar que estudos mostraram que as maiores concentrações da droga são encontradas no cérebro, no baço, nos rins e no pulmão ${ }^{(4)}$ e, portanto, estruturas corticais participantes do processamento dos estímulos auditivos podem estar comprometidas. Assim, sugere-se que estudos futuros utilizem Potenciais Evocados Auditivos de Média e Longa Latência para investigar a porção subcortical e cortical da via.

\section{CONCLUSÃO}

Não houve diferenças significativas dos potenciais evocados auditivos de tronco encefálico dos usuários de drogas quando comparados ao grupo controle.

As queixas apresentadas pelos usuários de drogas foram hiperacusia, alucinação auditiva, zumbido e alteração de equilíbrio. 


\begin{abstract}
Purpose: To study the findings of auditory brainstem evoked potentials in crack and multiple drugs users, as well as to raise possible auditory and balance complaints in this population. Methods: A total of 40 drugs addicts ( 20 who had been using drugs for over five years and 20 for less than five years) and 20 non-users were evaluated. Subjects were all male, with ages ranging from 19 to 46 years, and had auditory thresholds within normal. Results: No significant statistical differences were found regarding the auditory brainstem evoked potentials findings of the addicts group, when compared to the non-users. Conclusion: Auditory brainstem evoked potentials in crack and multiple drugs users did not differ significantly from the control group. Complaints presented by drugs addicts were hyperacusis, auditory hallucination, tinnitus and altered balance.
\end{abstract}

Keywords: Hearing; Auditory brain stem evoked potentials; Drug effects; Cocaine/adverse effects; Crack cocaine/adverse effects; Street drugs/adverse effects

\title{
REFERÊNCIAS
}

1. Ferreira PE, Martini RK. Cocaína: lendas, história e abuso. Rev Bras Psiquiatr 2001;23(2):96-9.

2. Winger G, Woods JH, Galuska CM, Wade-Galuska T. Behavioral perspectives on the neuroscience of drug addiction. J Exp Anal Behav. 2005;84(3):667-81.

3. Kessler FH, Woody G, Portela LV, Tort AB, De Boni R, Peuker AC et al. Brain injury markers (S100B and NSE) in chronic cocaine dependents. Rev Bras Psiquiatr. 2007;29(2):134-9.

4. Gazoni FM, Truffa AA, Kawamura C, Guimarães HP, Lopes RD, Sandre LV et al. Complicações cardiovasculares em usuário de cocaína. Relato de caso. Rev Bras Ter Intensiva. 2006;18(4):427-32.

5. Wang M. Perinatal drug abuse and neonatal drug withdrawal [Internet]. Omaha (NE): eMedicine; 2008; cited 2008 Jan 10. Available from: http://emedicine.medscape.com/article/978492-overview

6. Cone-Wesson B. Prenatal alcohol and cocaine exposure: influences on cognition, speech, language, and hearing. J Commun Disord. 2005;38(4):279-302.

7. Triqueiros-Cunha N, Leão P, Renard N, Tavares MA, Eybalin M. Prenatal cocaine exposure accelerates morphological changes and transient expression of tyrosine hydroxylase in the cochlea of developing rats. Brain Res. 2006;1086(1):55-64.

8. Shivapuja BG, Raya SM, Saunders SS, Schoenerc EP. Alpha 1-adrenergic receptor antagonist blocks acute cocaine action on the compound action potential of the auditory nerve in the chinchilla. Drug Alcohol Depend. 1995;40(1):43-8.

9. Malbergier A, Oliveira Jr H. Complicações médicas relacionadas ao uso da cocaína [Internet]. Rio de Janeiro: Associação Brasileira de Psiquiatria; 2006; citado 2006 Out 19. Disponível em: http:// www.abpbrasil.org.br/departamentos/coordenadores/coordenador/ noticias/?not $=139 \&$ dep $=62$

10. McLellan AT, Luborsky L, Woody GE, O'Brien CP. An improved diagnostic evaluation instrument for substance abuse patients. The addiction severity index. J Nerv Ment Dis. 1980;168(1):26-33.

11. Jerger J, Jerger S, Mauldin L. Studies in impedance audiometry. I. Normal and sensorineural ears. Arch Otolaryngol. 1972;96(6):513-23.
12. Ferreira Filho OF, Turchi MD, Laranjeira R, Castelo A. Perfil sociodemográfico e de padrões de uso entre dependentes de cocaína hospitalizados. Rev Saúde Pública. 2003;37(6):751-9.

13. Azevedo RC, Botega NJ, Guimarães LA. Crack users, sexual behavior and risk of HIV infection. Rev Bras Psiquiatr. 2007;29(1):26-30.

14. Duailibi LB, Ribeiro M, Laranjeira R. Profile of cocaine and crack users in Brazil. Cad Saúde Pública. 2008;24(Suppl 4):s545-57.

15. Jastreboff P, Jastreboff MM. Tinnitus Retraining Therapy (TRT) as a method for treatment of tinnitus and hyperacusis patients. J Am Acad Audiol. 2000;11(3):162-77.

16. Knobel KA, Sanchez TG. Hipersensibilidade auditiva. In: Samelli AG. Zumbido. Avaliação, diagnóstico e reabilitação: abordagens atuais. São Paulo: Lovise; 2004. p. 45-59.

17. Jastreboff P. Phantom auditory perception (tinnitus): mechanisms of generation and perception. Neurosci Res 1990;8(4):221-54.

18. Samelli AG. Zumbido. Avaliação, diagnóstico e reabilitação: abordagens atuais. São Paulo: Lovise; 2004. Hipóteses atuais sobre a geração do zumbido; p. 23-35.

19. Taguchi CK. Da funçäo vestibular em usuários de crack: pesquisa vectoeletronistagmográfica [tese]. São Paulo: Universidade Federal de São Paulo; 1997.

20. Hartmann M, Schmidt MH, Lay B, Blanz B, Cucchiaro G. Drogas ilícitas e esquizofrenia em adolescentes. Rev Psiquiatr Clín (São Paulo). 1999;26(3):62-7.

21. Moeller FG, Barratt ES, Fischer CJ, Dougherty DM, Reilly EL, Mathias $\mathrm{CW}$ et al. P300 event-related potential amplitude and impulsivity in cocaine-dependent subjects. Neuropsychobiology. 2004;50(2):167-73.

22. Boutros NN, Gooding D, Sundaresan K, Burroughs S, Johanson C. Cocaine-dependence and cocaine-induced paranoia and mid-latency auditory evoked responses and sensory gating. Psychiatry Res. 2006;145(2-3):147-54.

23. Gooding DC, Burroughs S, Boutros NN. Attentional deficits in cocainedependent patients: converging behavioral and electrophysiological evidence. Psychiatry Res. 2008;160(2):145-54. 
Anexo 1. Questionário adaptado do Protocolo ASI (Addiction Severity Index)

I - Histórico médico

- Quantas vezes você foi hospitalizado por problemas médicos (incluindo overdose e delirium)?

- Há quanto tempo foi sua última hospitalização por problemas clínicos?

- Você tem algum problema médico crônico que continua a interferir na sua vida?

- Você toma regularmente alguma medicação prescrita por médico para problemas físicos (excluindo medicamentos psiquiátricos)?

- Qual o grau de importância que o tratamento para estes problemas de saúde tem para você agora?

II - Histórico profissional e sustento

- Você tem uma profissão ou ocupação? Qual?

- Alguém contribui para o seu sustento, de alguma forma?

- Esta contribuição constitui a maior parte de seu sustento?

III - Informações sobre drogas e álcool (tempo de uso / abstinência / via de administração para cada uma destas substâncias)

- álcool

- heroína

- metadona

- opióides / analgésicos

- sedativos

- cocaína

- crack

- anfetaminas

- maconha

- alucinógenos

- inalantes

- Mais de uma substância por dia

- Vias de administração oral

nasal

fumada

injeção não endovenosa

injeção endovenosa

- Qual a substância que é o principal problema?

- Quanto tempo durou seu último período de abstinência voluntário para esta(s) substância(s) principal(ais)?

- Quantas vezes na vida você já foi tratado por este problema?

IV - Informações psicológicas

- Você teve um período significante em que apresentou:

- depressão?

- alucinação?

- problemas de compreensão, concentração ou memória? 